$02,05,08,10$

\title{
Динамические вихревые состояния в высокотемпературных сверхпроводниках при импульсном намагничивании
}

\author{
(C) А.Н. Мороз, А.Н. Максимова, В.А. Кашурников, И.А. Руднев \\ Национальный исследовательский ядерный университет „МИФИ“, \\ Москва, Россия \\ E-mail: iarudnev@mephi.ru
}

Поступила в Редакцию 30 декабря 2019 г.

В окончательной редакции 30 декабря 2019 г.

Принята к публикации 10 января 2020 г.

\begin{abstract}
Методом Монте-Карло проведено численное моделирование вихревой системы сверхпроводника Вi-2212 в линейно нарастающем внешнем магнитном поле для разных скоростей роста. Исследовано влияние концентрации и распределения центров пиннинга на проникновение магнитного потока. Обнаружены особенности проникновения потока и описаны с точки зрения динамики вихревой системы, определены условия их возникновения. Получены растущие зависимости величины поля насыщения от концентрации дефектов для однородных и неоднородных образцов.
\end{abstract}

Ключевые слова: ВТСП, пиннинг, вихри Абрикосова, импульсное намагничивание, метод Монте-Карло.

DOI: $10.21883 /$ FTT.2020.05.49226.25M

\section{1. Введение}

Современные высокотемпературные сверхпроводники (ВТСП) являются объектами повышенного интереса благодаря возможности их использования в качестве магнитов с захваченным магнитным потоком [1-17]. Их преимущество перед обычными магнитами (например, $\mathrm{NdFeB}$ ) заключается в том, что намагниченность ВТСП определяется не только внутренними свойствами материала, но и его размерами, и это позволяет увеличить захваченный магнитный поток и получить более сильные поля. Как правило, на практике используют объемные ВТСП в форме шайбы (pellet) $[1,2]$ или стопки из ВТСП-лент [3-6].

На сегодняшний день применяются два основных метода намагничивания ВТСП: статический и импульсный. Статические методы, а именно охлаждение в поле (FC — field cooling), и в нулевом поле (ZFC — zero field cooling) пока позволяют получать более высокие захваченные поля по сравнению с импульсным намагничиванием. Так, в работе [7] продемонстрирована возможность захвата магнитного поля в $17.6 \mathrm{~T}$ при $T=26 \mathrm{~K}$. Однако процесс намагничивания занимает продолжительное время и является дорогостоящим, так как требует использования высокопольных сверхпроводящих магнитов или магнитов Биттера [6]. Импульсным методам намагничивания сегодня уделяется много внимания благодаря их относительно невысокой стоимости, компактности и высокой скорости намагничивания [8]. Тем не менее данным методом до сих пор не удалось получить захваченное магнитное поле больше 6 Т при тех же температурах, во многом из-за локального перегрева образца, вызванного быстрым движением вихрей Абрикосова в условиях сильно неравновесного состояния магнитного потока в сверхпроводнике при импульсном нарастании внешнего магнитного поля $[9,10]$. Для снижения влияния перегрева и повышения величины захваченного поля выполнен ряд экспериментальных исследований по подбору температурных режимов [11], методов приложения импульсов магнитного поля [12-13], типов и параметров катушек [14].

Для описания процессов захвата магнитного потока в высокотемпературных сверхпроводниках при импульсном намагничивании используются феноменологические подходы, позволяющие провести моделирование намагничивания методом конечных элементов в различных физических формулировках [15-17]. Вместе с тем к настоящему времени в литературе практически отсутствуют теоретические исследования поведения вихревой системы под действием импульсов магнитного поля и его влияния на процессы намагничивания. В связи с этим целью настоящей работы является изучение неравновесной динамики вихрей Абрикосова под действием быстро нарастающего внешнего магнитного поля.

\section{2. Расчетная модель}

Моделирование системы вихрей Абрикосова проводилось континуальным методом Монте-Карло в рамках двумерной модели слоистого высокотемпературного сверхпроводника [18-20]. Данный подход показал свою эффективность и ранее применялся нашим коллективом для исследования влияния на транспортные и магнитные свойства ВТСП дефектов различного типа (точечных, протяженных, ферромагнитных) [21-23], анизотропии [24], деформаций изгиба [25] и искусственных дефектов в виде микроскопических отверстий [26]. Ме- 
тод позволяет учитывать все возможные взаимодействия вихревой системы, в том числе с различными центрами пиннинга, рассчитывать кривые намагниченности и вольт-амперные характеристики различных ВТСПматериалов, равновесные и неравновесные состояния вихревой системы.

В двумерном ВТСП вихри Абрикосова можно представить в виде их проекций на плоскость - плоских „блинов“ (pancakes). Термодинамический потенциал Гиббса $G$ системы из $N$ вихрей на единицу толщины сверхпроводящего слоя можно представить в виде (1):

$$
\begin{aligned}
G= & \sum_{i<j} U_{\mathrm{vv}}\left(\mathbf{r}_{i j}\right)+\sum_{i, j} U_{\text {surf }}\left(\left|\mathbf{r}_{i}-\mathbf{r}_{j}^{\mathrm{img}}\right|\right) \\
& +\sum_{i, j} U_{\text {pin }}\left(\left|\mathbf{r}_{i}-\mathbf{r}_{j}^{\mathrm{def}}\right|\right)+\sum_{i} U_{m}\left(\mathbf{r}_{i}\right)+N \varepsilon
\end{aligned}
$$

Первое слагаемое в нем отвечает за межвихревое отталкивательное взаимодействие (,vv“ = vortex-vortex), далее следуют: взаимодействие с поверхностью образца (которое представляется в виде взаимодействия вихря $\mathbf{r}_{i}$ с изображениями всех вихрей системы $\mathbf{r}_{j}^{\mathrm{img}}$ относительно границы), с центрами пиннинга (дефектами $\mathbf{r}_{j}^{\text {def }}$ ), с мейсснеровским током, а также сумма собственных энергий вихрей $\varepsilon$. Дефекты в рамках модели представляются в виде локальных потенциальных ям глубиной $\alpha$ и шириной $\sim 2 \xi$, где $\xi-$ длина когерентности. Суть алгоритма Монте-Карло (МК) заключается в минимизации потенциала Гиббса системы для конкретных внешних условий системы (температуры и магнитного поля) через процессы рождения, уничтожения и движения случайно выбранных вихрей.

Объектом исследований в настоящей работе был сверхпроводник $\mathrm{Bi}_{2} \mathrm{Sr}_{2} \mathrm{CaCu}_{2} \mathrm{O}_{8+x}$ (Bi-2212), для которого были использованы следующие параметры, известные из эксперимента (ссылка 14 в работе [19]): $\lambda=180 \mathrm{~nm}$ - глубина проникновения магнитного поля, $T_{c}=84 \mathrm{~K}-$ критическая температура, $\xi=2 \mathrm{~nm}$. Образцы содержали различное число центров пиннинга в двух пространственных конфигурациях: случайное распределение и треугольная решетка. Треугольная решетка дефектов была выбрана для изучения однородности проникновения магнитного потока в образец с равномерно распределенными центрами пиннинга и сравнения результатов с неоднородным (случайным) распределением такого же числа дефектов. Общее количество дефектов в образце менялось от 0 до 1000. Образец имел форму квадрата со стороной $5 \mu \mathrm{m}$ с периодическими граничными условиями по оси $y$ и жесткой границей по оси $x$, через которую происходило проникновение вихрей в образец. Такой выбор геометрии позволяет моделировать бесконечную ВТСП-ленту. Расчеты производились для температуры $T=1 \mathrm{~K}$ в отсутствие транспортного тока.

Внешнее магнитное поле, перпендикулярное плоскости образцов, менялось в диапазоне $\sim 400-1500$ Ое с пятью различными скоростями роста. Для этого каждые $10^{4}$ элементарных шагов Монте-Карло внешнее магнитное поле увеличивалось на фиксированную величину $(1.0,2.5,5.0,7.5,10.0 \mathrm{Oe})$. Ввиду отсутствия реального времени в расчете, величина $10^{4}$ шагов МК была выбрана как наименьший временной промежуток, за который в вихревой системе устанавливается динамическое равновесие. Общее время расчета при этом составило от $\sim 10^{6}$ (для инкремента магнитного поля в $10.0 \mathrm{Oe} / 10^{4}$ шагов) до $\sim 10^{7}$ шагов Монте-Карло (для инкремента в $1.0 \mathrm{Oe} / 10^{4}$ шагов).

Расчет нестационарных процессов в рамках нашего метода возможен благодаря эквивалентности среднего по ансамблю среднему по времени при достаточно большом количестве отсчетов: в процессе моделирования физический интерес представляют только конечные состояния вихрей, а не их отдельные перемещения, однако метод позволяет проследить за эволюцией системы в эти конечные состояния. При этом каждому шагу можно поставить в соответствие реальное время путем нормировки на эксперимент. По нашим оценкам, $10^{6}$ шагов МК эквивалентны $\sim 10^{-4} \mathrm{~s}$, что соответствует характерным временам релаксации в ВТСП.

\section{3. Результаты моделирования}

На рис. 1 представлены временные зависимости усредненного по площади образца магнитного поля $B$ внутри сверхпроводника со 165 дефектами, что соответствует двумерной плотности $n_{d} \sim 6 \cdot 10^{8} \mathrm{~cm}^{-2}$. В одном образце дефекты были распределены случайно („random“, слева), в другом - выстроены в треугольную решетку („triangular", справа) с параметром $a=400 \mathrm{~nm}$. Здесь $B$ - интегральная характеристика, эквивалентная общей величине магнитного потока в образце (один из важных параметров для оценки качества ВТСП-магнита [13]), и рассчитывается как $N \Phi_{0} / S$, где $\Phi_{0}=2.07 \cdot 10^{7} \mathrm{G} \cdot \mathrm{cm}^{2}$ — квант магнитного потока, который несет в себе каждый вихрь, $S=25 \mu \mathrm{m}^{2}-$ площадь образца. На всех подобных рисунках (именуемых также магнитными откликами образца) далее символами обозначено магнитное поле $B$ внутри образца, а линиями - внешнее магнитное поле $H$. Под временными зависимостями далее будут подразумеваться зависимости от номера шага Монте-Карло $N_{\mathrm{MC}}$.

На рис. 1 видно, что в случае ,random“ магнитное поле внутри образца нарастает линейно и с той же скоростью, что внешнее поле. При этом данная зависимость наблюдается для всех пяти скоростей роста $H$, а конечные величины $B$ для них практически совпадают. Стоит отметить, что почти идентичная ситуация наблюдалась и для бездефектного образца, т. е. случай 165 случайно распределенных дефектов отвечает образцу со слабым пиннингом. Случай „triangular“ демонстрирует ступенчатые особенности: в начале действия внешнего поля $B$ меняется нелинейно, претерпевает три небольших скач- 


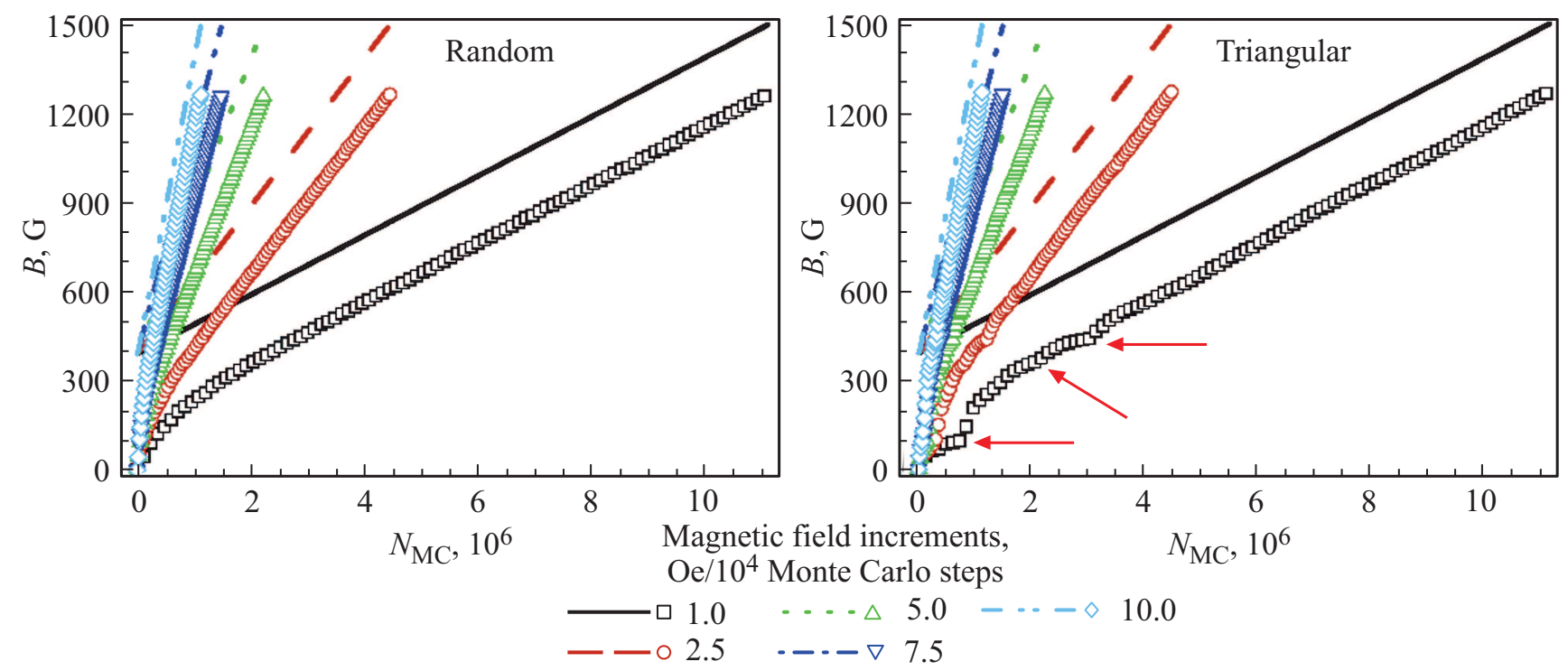

Рис. 1. Временны́е зависимости усредненного поля $B$ внутри сверхпроводника (символы) для образцов со 165 дефектами, распределенными случайно (слева) и выстроенными в треугольную решетку (справа), для разных скоростей нарастания внешнего поля. Стрелками отмечены скачкообразные особенности на зависимостях.

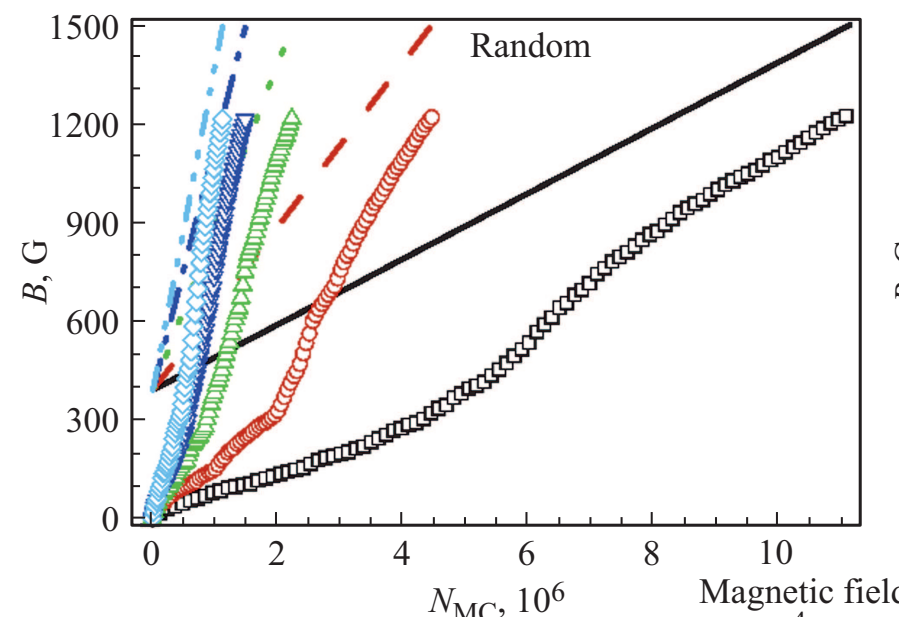

$0 \mathrm{e} / 10^{4} \mathrm{M}$

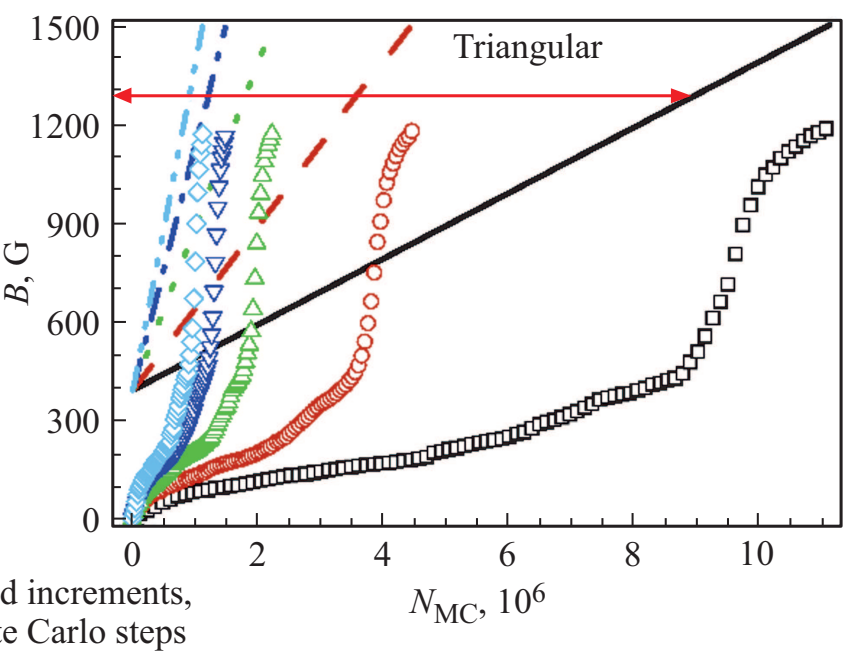

$\longrightarrow \square 1.0 \quad \cdots \triangle 5.0 \quad \cdots-\diamond 10.0$

Рис. 2. Временны́е зависимости усредненного поля $B$ внутри сверхпроводника (символы) для образцов с 1000 дефектов, распределенных случайно (слева) и выстроенных в треугольную решетку (справа), для разных скоростей нарастания внешнего поля. Горизонтальная стрелка указывает на величину $H$, при которой наблюдается скачок $B$.

ка (отмечены стрелками), и затем после $\sim 3 \cdot 10^{6}$ шагов МК выходит на линейный участок, параллельный изменению внешнего поля. Подобные скачки наблюдаются для всех кривых на рисунке и при тех же величинах внешнего поля (в случае большого инкремента они менее заметны на фоне общего роста поля).

В сильно дефектном случае (1000 дефектов, $\left.n_{d} \sim 4 \cdot 10^{9} \mathrm{~cm}^{-2}, a=160 \mathrm{~nm}\right)$, продемонстрированном на рис. 2, нелинейный отклик на внешнее поле демонстрирует уже образец „random“. А именно, в первую половину времени нарастания $H$ поле внутри образца меняется медленнее, чем внешнее, затем наблюдается плавный переходный участок длительностью около $\sim 2.5 \cdot 10^{6}$ шагов МК, после чего поле внутри начинает меняться параллельно внешнему полю. Отклик образца „triangular“ большую часть времени расчета $\left(\sim 9 \cdot 10^{6}\right.$ шагов) нелинеен (причем рост магнитного поля внутри образца происходит медленнее, чем в случае „random“), после чего наблюдается резкий (за время меньше или порядка $10^{6}$ шагов МК) скачок большой амплитуды ( $600 \mathrm{Oе})$, и затем переход к линейному участку. Отметим, что данный скачок происходит при практи- 

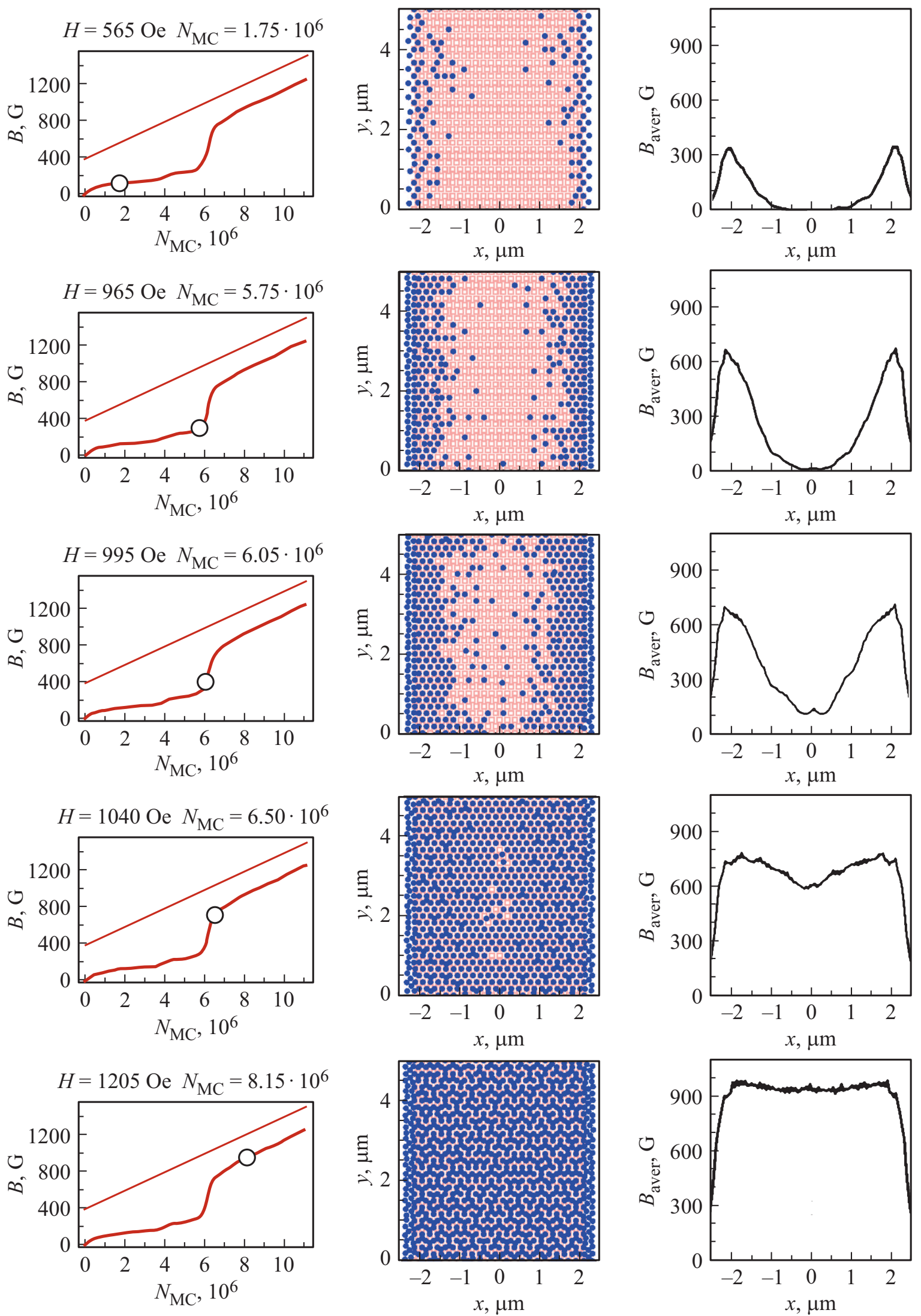

Рис. 3. Временна́я зависимость магнитного поля внутри образца (слева), мгновенные вихревые конфигурации (посередине) и усредненные профили распределения индукции поля внутри образца (справа) в разные моменты времени нарастания $H$ со скоростью $1 \mathrm{Oe} / 10^{4}$ шагов МК для образца с треугольной решеткой 700 дефектов. 
чески одной и той же величине внешнего поля (показано стрелкой), независимо от скорости его нарастания.

Рассмотрим динамику вихревой системы и особенности ее отклика на намагничивание на примере образца с треугольной решеткой 700 дефектов $\left(n_{d} \sim 3 \cdot 10^{9} \mathrm{~cm}^{-2}\right.$, $a=192 \mathrm{~nm})$ при изменении внешнего магнитного поля со скоростью $1.0 \mathrm{Oe} / 10^{4}$ шагов Монте-Карло. На рис. 3 представлены: временна́я зависимость $B$ с указанием различных моментов времени (слева), мгновенные вихревые конфигурации в обозначенные моменты времени (посередине) и усредненные по высоте образца профили распределения индукции магнитного поля $B_{\text {aver }}$ внутри образца в те же моменты времени (справа). $B_{\text {aver }}$ также является интегральной характеристикой, определяемой суммированием создаваемых всеми вихрями магнитных полей в каждой точке сверхпроводника.

В самом начале действия внешнего магнитного поля входящие в образец через границу вихри оказываются закрепленными на ближайших дефектах (момент времени, соответствующий $H=565 \mathrm{Oe})$. Далее по мере роста внешнего поля происходит накапливание вихрей вблизи границы $(H=965 \mathrm{Oe})$, в то время как в центре образца магнитное поле отсутствует - профиль $B_{\text {aver }}$ имеет провал в центре образца и максимумы по краям. По достижении внешним магнитным полем некоторой пороговой величины происходит быстрое заполнение вихрями сначала дефектов, находящихся в центре образца, а затем - промежутков между ними, что приводит к выравниванию профиля $B_{\text {aver }}$ от границы к центру (моменты времени $H=995 \mathrm{Oe}$ - начало перехода и $H=1040$ Ое - конец перехода). Одновременно с этим наблюдается резкий скачок на зависимости $B\left(N_{\mathrm{MC}}\right)$. Момент времени выравнивания профиля $B_{\text {aver }}$ соответствует ситуации, при которой все дефекты в образце оказываются занятыми вихрями.

При дальнейшем увеличении внешнего магнитного поля новые вихри, входящие в сверхпроводник, заполняют свободное пространство между дефектами. Как можно видеть из мгновенных вихревых конфигураций, сначала происходит образование треугольных кластеров, в которых свободный вихрь располагается в геометрическом центре правильного треугольника, составленного из закрепленных дефектами вихрей $(H=1040$ Ое), затем появляются группы таких кластеров, а после формируется треугольная решетка вихрей с периодом, примерно в два раза меньшим, чем период решетки дефектов (момент времени $H=1205 \mathrm{Oе}$ ). После того как все дефекты оказались занятыми и профиль индукции поля внутри образца выровнялся, количество вихрей в сверхпроводнике становится пропорциональным величине внешнего поля, в результате чего $B$ растет практически линейно и с той же скоростью, что $H$. При этом оказалось, что чем больше дефектов содержится в образце (в пределах рассмотренных в настоящей работе значений), тем выше пороговая величина внешнего магнитного поля, при которой наблюдается скачок $B$.

\section{4. Обсуждение результатов}

Проникновение магнитного потока в ВТСП определяется не только величиной внешнего магнитного поля, но и потенциалом пиннинга образца. Как известно, вихри одного знака отталкиваются, поэтому в условиях равномерно распределенного вблизи границы потенциала пиннинга (как в случае треугольной решетки дефектов) закрепленные вихри формируют приграничный потенциальный барьер [19], ограничивающий движение и проникновение магнитного потока в образец при увеличении внешнего поля. Высота этого барьера определяется глубиной потенциальной ямы дефектов (в наших расчетах она составляла $0.05 \mathrm{eV}$ ). Таким образом, вблизи границы на закрепленный вихрь действует сила пиннинга, а также равнодействующая сил отталкивания со стороны ближайших вихрей и силы Лоренца со стороны Мейсснеровского тока, стремящаяся продвинуть вихрь к центру образца. Как только эта равнодействующая сравнивается с силой пиннинга, приграничный барьер разрушается и происходит быстрое заполнение образца магнитным потоком, причем вихри занимают наиболее энергетически выгодные положения. В случае треугольной решетки дефектов таким положением оказывается вложенная в нее треугольная решетка вихрей с меньшим периодом. Можно ожидать, что по мере дальнейшего увеличения внешнего магнитного поля (за пределами рассмотренного в настоящей работе) произойдет еще один скачок на временной зависимости захваченного магнитного поля образца с 700 дефектов, но уже меньшей амплитуды - когда вложенная решетка окажется заполненной и произойдет перестройка конфигурации вихрей. Похожее явление привело к последовательности из трех малых скачков на такой же зависимости для образца „triangular“ со 165 дефектами (рис. 1). Увеличение концентрации дефектов приводит к повышению эффективности приграничного потенциального барьера, в результате чего пороговое значение внешнего магнитного поля увеличивается.

Случайное распределение дефектов отличается от треугольной решетки наличием локальных скоплений центров пиннинга (кластер дефектов) и, соответственно, локальных пустот. Наличие таких неоднородностей приводит к тому, что, во-первых, внутри образца образуются каналы для свободного движения вихрей, а во-вторых, не все дефекты в кластере могут выступить в качестве центров пиннинга. Последний эффект наблюдается в том случае, когда из-за сильного отталкивания между вихрями из нескольких близко расположенных дефектов в кластере вихрем занят только один. В результате приграничный потенциальный барьер оказывается гораздо менее эффективным, чем при однородном распределении дефектов, и продвижение вихрей к центру образца оказывается возможным при меньших магнитных полях. При этом он происходит не скачкообразно, как в случае треугольной решетки, а плавно (рис. 2). Если количество дефектов невелико (например, в случае рис. 1), магнит- 


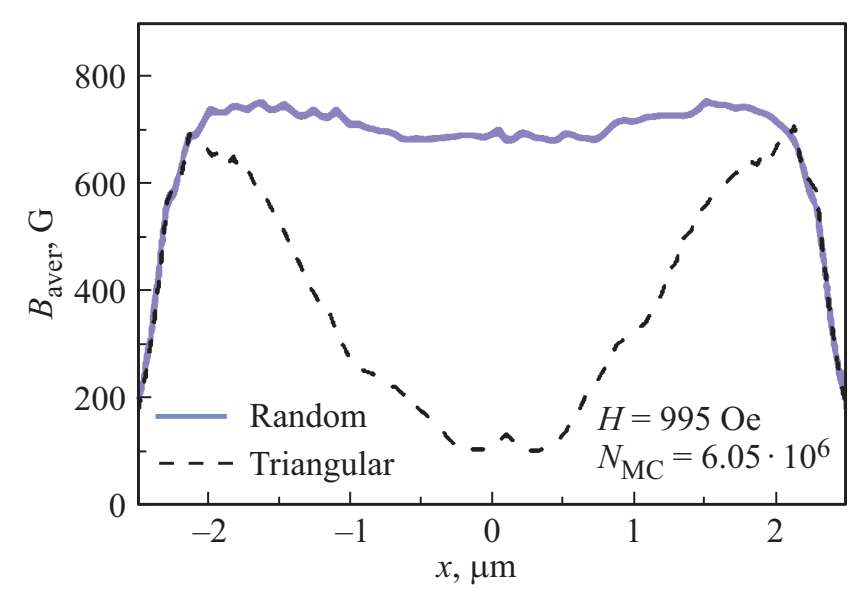

Рис. 4. Усредненные профили индукции магнитного поля внутри образцов с 700 дефектов, распределенных случайно и выстроенных в треугольную решетку, в одном и том же магнитном поле.

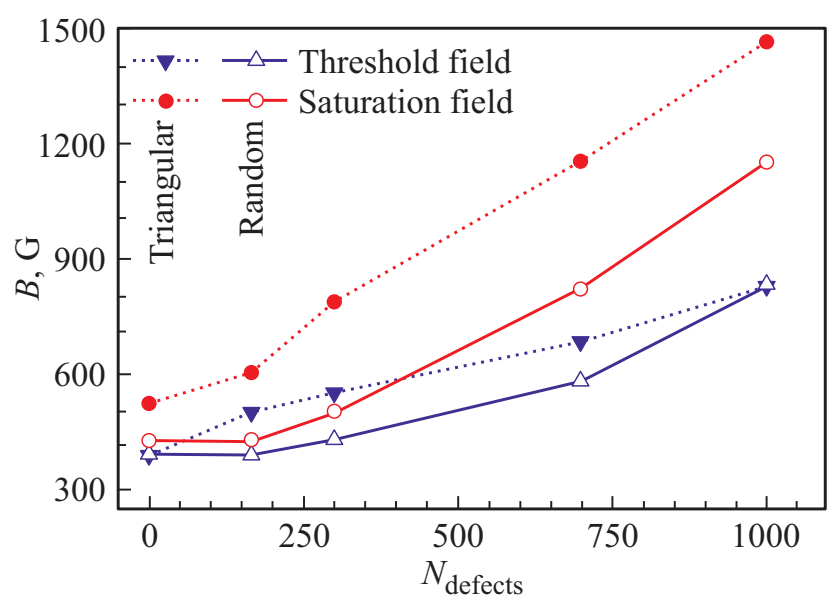

Рис. 5. Зависимости поля насыщения и порогового поля от числа дефектов для случайного и равномерного распределения.
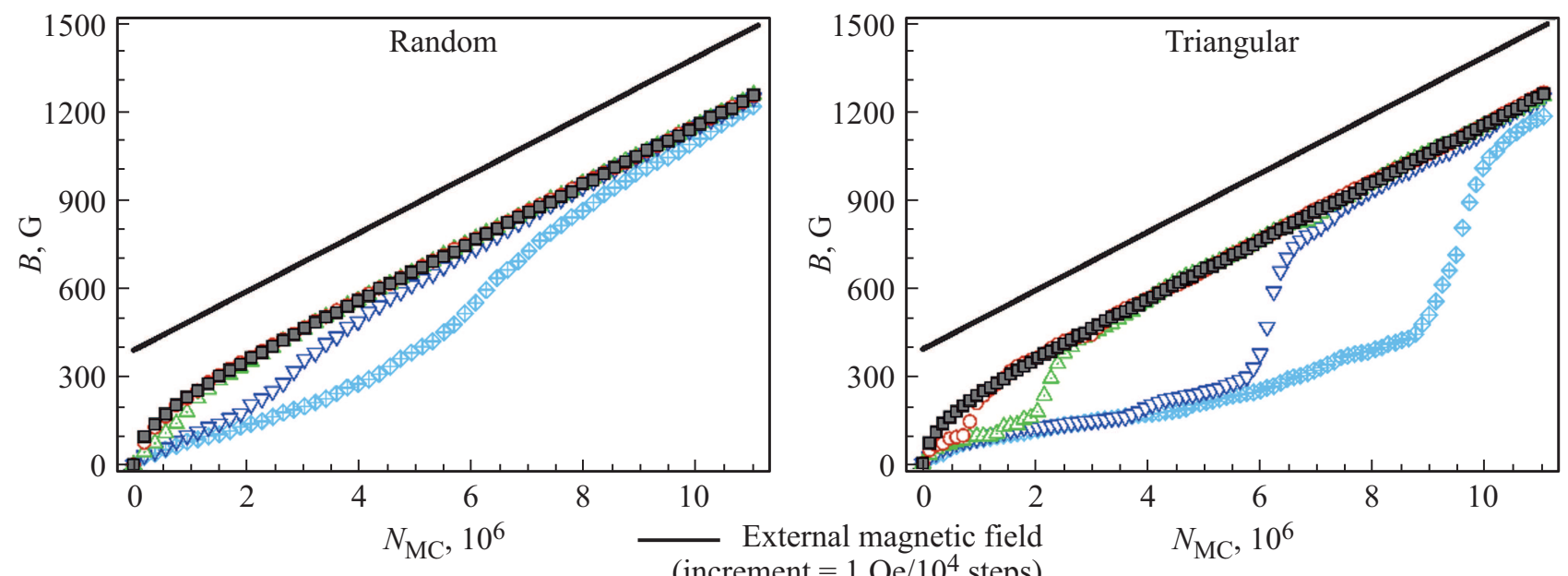

(increment $=1 \mathrm{Oe} / 10^{4}$ steps)

Integral magnetic field, different numbers of defects:
$\square 0$
○ 165
$\triangle 300$
$\nabla 700$
1000

Рис. 6. Временнб́е зависимости усредненного магнитного поля внутри образцов „random“ и „triangular“ для разного числа дефектов.

Физика твердого тела, 2020, том 62, вып. 5 
ленных на дефектах вихрей. Ввиду того, что при случайном распределении дефектов меньше вихрей окажется закрепленными, можно предположить, что и остаточный захваченный магнитный поток в этом случае будет ниже.

На рис. 5 представлены зависимости полей насыщения и пороговых полей (имеется в виду поле, при котором начинается переход от нелинейного участка временной зависимости $B\left(N_{\mathrm{MC}}\right)$ к линейному) от концентрации дефектов для образцов „random“ и „triangular“. В пределах рассмотренных концентраций и магнитных полей наблюдается рост обеих величин, причем с увеличением числа дефектов поле насыщения увеличивается относительно порогового, т.е. растет амплитуда перехода от нелинейного участка магнитного отклика к линейному. Это можно объяснить растущей эффективностью пиннинга - большее число вихрей накапливается на границе до момента выравнивания силы пиннинга и равнодействующей сил взаимного отталкивания вихрей и силы Лоренца.

Участки нелинейности магнитных откликов образцов с треугольной решеткой дефектов лежат примерно на одной линии (рис. 6, справа). Этот эффект можно объяснить тем, что вихревая система (которая в абсолютно бездефектном случае выстраивается в треугольную решетку Абрикосова) подстраивается под треугольную решетку дефектов при формировании приграничного барьера, занимая наиболее энергетически выгодное положение при данной величине внешнего магнитного поля. Минимум энергии определяется, в первую очередь, величиной $H$ и потенциалом взаимодействия вихрей друг с другом, а дефекты только вносят вклад в перераспределение вихрей вблизи границы. В результате количество вихрей в образцах с разным числом дефектов при одних и тех же величинах внешнего поля (ниже порогового значения для каждого конкретного образца) оказывается одинаковым. В образцах со случайным распределением дефектов наблюдается похожая ситуация, но в меньшем масштабе (рис. 6, слева). Для них этот эффект вызван тем, что при генерации координат дефектов стартовое случайное число сохранялось. Поэтому, например, в числе 1000 дефектов содержались те же дефекты, что в образце с 700, и 300 дополнительных.

\section{5. Заключение}

В настоящей работе проведено численное исследование динамики вихревой системы слоистого высокотемпературного сверхпроводника под действием быстрого линейного намагничивания. Обнаружены особенности проникновения магнитного потока в образцы с равномерным распределением потенциала пиннинга в виде скачков усредненного по площади образца магнитного поля внутри сверхпроводника, зависящих от концентрации дефектов. Показано, что это явление связано c формированием приграничного барьера из закрепленных дефектами вихрей и накоплением магнитного потока на краях образца, а затем - разрушением этого барьера с превышением внешнего магнитного поля некоторого порогового значения. На основании обнаруженных особенностей определены величины поля насыщения и их зависимость от количества дефектов в образце: в пределах рассмотренных концентраций дефектов и максимальной величины внешнего поля данные зависимости оказались растущими, в том числе относительно порогового поля. Показано, что неоднородное распределение центров пиннинга приводит к снижению поля насыщения. Результаты расчетов качественно соответствуют литературным экспериментальным данным.

\section{Финансирование работы}

Исследование выполнено при финансовой поддержке РФФИ в рамках научного проекта № 17-29-10024.

\section{Конфликт интересов}

Авторы заявляют, что у них нет конфликта интересов.

\section{Список литературы}

[1] M.D. Ainslie, H. Fujishiro, T. Ujiie, J. Zou, A.R. Dennis, Y.-H. Shi, D.A. Cardwell. Supercond. Sci. Technol. 27, 065008 (2014).

[2] T. Ida, Z. Li, D. Zhou, M. Miki, Y. Zhang, M. Izumi. Supercond. Sci. Technol. 29, 054005 (2016).

[3] A. Patel, S.C. Hopkins, B.A. Glowacki. Supercond. Sci. Technol. 26, 032001 (2013).

[4] I. Rudnev, D. Abin, M. Osipov, S. Pokrovskiy, Y. Ermolaev, N. Mineev. Phys. Procedia 65, 141 (2015).

[5] N. Mineev, I. Rudnev. IEEE Trans. Appl. Supercond. 26, 8200904 (2016).

[6] K. Takahashi, M.D. Ainslie, H. Fujishiro, T. Naito, Y-H. Shi, D.A. Cardwell. Physica C 536, 1 (2017).

[7] J.H. Durrell, A.R. Dennis, J. Jaroszynski, M.D. Ainslie, K.G.B. Palmer, Y-H. Shi, A.M. Campbell, J. Hull, M. Strasik, E.E. Hellstrom, D.A. Cardwell. Supercond. Sci. Technol. 27, 082001 (2014).

[8] Z. Deng, N. Shinohara, M. Miki, B. Felder, K. Tsuzuki, M. Watasaki, S. Kawabe, R. Taguchi, M. Izumi. Phys. Procedia 36, 958 (2012).

[9] H. Fujishiro, M. Kaneyama, K. Yokoyama, T. Oka, K. Noto. Supercond. Sci. Technol. 18, 158 (2005).

[10] H. Fujishiro, M. Kaneyama, T. Tateiwa, T. Oka. J. Phys. Conf. Ser. 43, 405 (2006).

[11] M.D. Ainslie, J. Srpcic, D. Zhou, H.Fujishiro, K. Takahashi, D.A. Cardwell, J.H. Durrell. IEEE Trans. Appl. Supercond. 28, 6800207 (2018).

[12] H. Fujishiro, T. Tateiwa, A. Fujiwara, T. Oka, H. Hayashi. Physica C 445-448, 334 (2006).

[13] Z. Deng, M. Miki, K. Tsuzuki, B. Felder, R. Taguchi, N. Shinohara, M. Izumi. IEEE Trans. Appl. Supercond. 21, 1180 (2011).

[14] K. Berger, J. Kapek, A. Colle, M. Stepien, B. Grzesik, T. Lubin, J. Lévêque. IEEE Trans. Appl. Supercond. 28, 6801205 (2018). 
[15] J. López, R. Maynou, X. Granados, R. Bosch, J. Grau. IEEE Trans. Appl. Supercond. 23, 4901104 (2013).

[16] Z. Huang, H.S. Ruiz, T.A. Coombs. Physica C 534, 73 (2017).

[17] J. Arnaud, J.F.P. Fernandes, P.J.C. Branco. IEEE Trans. Appl. Supercond. 28, 6801604 (2018).

[18] В.А. Кашурников, И.А. Руднев, М.Е. Грачева, О.А. Никитенко. ЖЭТФ 117, 196 (2000).

[19] В.А. Кашурников, И.А. Руднев, М.В. Зюбин. ЖЭТФ 121, 442 (2002).

[20] S. Ryu, S. Doniach, G. Deutscher, A. Kapitulnik. Phys. Rev. Lett. 68, 710 (1992).

[21] V.A. Kashurnikov, A.N. Maksimova, I.A. Rudnev, D.S. Odintsov. Physica C 528, 17 (2016).

[22] В.А. Кашурников, А.Н. Максимова, И.А. Руднев, Д.С. Одинцов. ФТТ 57, 1685 (2015).

[23] В.А. Кашурников, А.Н. Максимова, И.А. Руднев. ФТТ 56, 861 (2014).

[24] В.А. Кашурников, А.Н. Максимова, И.А. Руднев. Д.С. Одинцов. ФТТ 58, 1457 (2016).

[25] V.A. Kashurnikov, A.N. Maksimova, A.N. Moroz, I.A. Rudnev. Supercond. Sci. Technol. 31, 115003 (2018).

[26] A.N. Moroz, A.N. Maksimova, V.A. Kashurnikov. I.A. Rudnev. IEEE Trans. Appl. Supercond. 28, 8000705 (2018).

Редактор Ю.Э. Китаев 